\title{
Diabetes en la era preinsulínica
}

\section{Alfredo Jácome Roca*}

${ }^{*} \mathrm{MD}$, FACP. Internista-endocrinólogo, miembro honorario de la Asociación Colombiana de Endocrinología, Diabetes y Metabolismo, miembro de número de la Academia Nacional de Medicina y miembro activo de la Sociedad Colombiana de Historia de la Medicina, Bogotá.

Fecha de recepción: 5/10/2016

Fecha de aceptación: 26/10/2016

Desde la antigüedad se habló de la diabetes. Se hicieron descripciones aisladas a menudo desconocidas por posteriores investigadores, no relacionaban su hallazgo con la enfermedad y daban explicaciones incorrectas sobre la causa de lo que observaban.

\section{Descubrimiento de la insulina}

La insulina fue descubierta en la ciudad de Toronto en el verano del año 1921, gracias a la tenacidad de un médico que logró el importante hallazgo científico. En 1920, Frederick Banting (1891-1941), en la revista Surgery, Gynecology \& Obstetrics encontró un caso autopsiado de Moses Barron sobre litiasis pancreática con atrofia de los acinis y persistencia de los islotes, parecido a lo que se observaba al bloquear por ligaduras un conducto pancreático ${ }^{(1)}$. Esa noche escribió: “Diabetes. Ligar el conducto pancreático del perro. Mantener los perros vivos hasta que se degeneren sus acinis, quedando los islotes. Tratar de aislar la secreción interna de estos para aliviar la glucosuria".

Figura 1. Charles Best y Frederick Banting

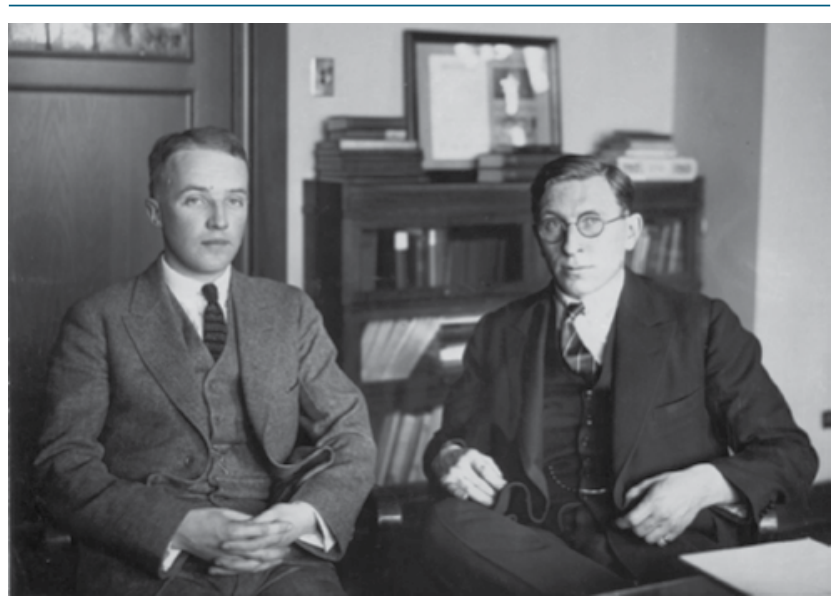

El joven Banting, con experiencia quirúrgica como ortopedista, acudió a John McLeod (1876-1935), fisiólogo de la Universidad de Toronto, para exponerle su idea y pedirle ayuda, pues en su universidad local no había recursos para hacerlo. En 1921, McLeod le prestó su laboratorio con algunos perros al joven Charley Best (1899-1990), como asistente (figura 1), con un grado de Baccalaureate en fisiología y bioquímica. No era mucho pero así se gestó el descubrimiento de la insulina. Los canadienses lograron hacer un extracto del páncreas que se atrofió al ligársele el conducto de Wirsung; tajadas de él fueron colocadas en solución de Ringer, enfriadas y maceradas en mortero y luego filtradas. Una hora después de inyectada la solución a un Terrier diabético, la glucemia descendió para volver a subir después de pasar azúcar por una sonda nasogástrica, aunque ni la hiperglucemia ni la glicosuria fueron tan marcadas como sucedió con un perro al que, sin darle el extracto, se le había hecho previamente esto. Los dos investigadores habían hecho un descubrimiento extraordinario ¡Era el nacimiento de la insulina! $\mathrm{Al}$ año siguiente, sus investigaciones fueron publicadas en el Canadian Medical Asociation Journal(2-4).

\section{Los que casi consiguen el crédito del descubrimiento}

Desde antes de Banting se pensaba que en el páncreas debía haber una hormona, que Meyer en 1909 había denominado insulina. Primero se realizaron los experimentos de Minkowski, que comprobaron la diabetes en perros sometidos a pancreatectomías, pero el teutón no pudo conseguir un preparado pancreático efectivo. Luego, Opie y Sobolev habían afirmado que los islotes pancreáticos eran necesarios para el control metabólico de los carbohidratos, y que la patología de estas células era la responsable de la diabetes.

El internista Georg Ludwig Zuelzer había preparado un extracto pancreático que administró a perros con glucosuria causada por epinefrina, e incluso a algunos pacientes, observando algunos efectos hipoglucemiantes, pero dicho extracto resultó tóxico por ser a base de alcohol. El fisiólogo Marcel Eugène Gley había depositado una carta sellada en la Sociedad de Biología de París, para abrirla hasta que él lo ordenara. Allí afirmaba que con un extracto a base de los restos atróficos de páncreas de conducto ligado, había disminuido la glucosuria 
de un perro pancreatectomizado. Se dice que no tenía confianza ciega en sus investigaciones.

El rumano Nicolae Paulesco había obtenido observaciones parecidas ${ }^{(5)}$. El caso de este fisiólogo de Bucarest es, al parecer, más injusto. Los rumanos protestaron fuertemente por la adjudicación del Nobel de Medicina por el descubrimiento de la insulina a los canadienses, pues consideraban que primero había hecho el descubrimiento su compatriota. En 1916 Paulesco tuvo éxito en el desarrollo de un extracto pancreático acuoso que normalizaba la glucemia en los perros diabéticos, pero no pudo continuar sus experimentos debido a la primera gran conflagración europea. Pero al finalizar la guerra, continuó con sus investigaciones y aisló la pancreína, su versión de la insulina. Dicen sus defensores que entre abril y junio de 1921, Paulesco presentó cuatro trabajos ante la sección rumana de la Sociedad de Biología de París (que resumían sus investigaciones) y luego logró publicar en el número de agosto de 1921 de la revista belga Archives Internationelles de Physiologie un extenso artículo sobre el papel del páncreas en la asimilación de los alimentos. En abril de 1922 consiguió una patente del gobierno rumano para la fabricación de la pancreína, pero estos hechos fueron ignorados por la comunidad internacional. Intentos posteriores de dar crédito a los trabajos del rumano se han visto frenados por razones políticas, ya que el fisiólogo consideraba que había un complot judeo-masónico contra la nación rumana.

\section{El metabolismo intermediario}

El desarrollo de la bioquímica en la primera mitad del siglo XX se debió a una serie de científicos de Europa y de los Estados Unidos, quienes en su mayoría recibieron el Premio Nobel, bien de Química, de Medicina o ambos. En 1902, Hermann E. Fischer demostró que las proteínas estaban compuestas de aminoácidos, unidas por un enlace peptídico, concluyendo más tarde que la acción de una enzima era específica en relación con un substrato. Warburg descubrió el sistema de citocromos que contienen hierro. Hill y Meyerhof hicieron estudios sobre el metabolismo muscular; este último, con Gustav Embden, investigó la transformación del glucógeno en ácido láctico. A fines de los años veinte, los bioquímicos estaban de acuerdo en que los ácidos fosfóricos tenían una importancia decisiva en la producción de la energía muscular. Después vendrían los Cori, Sanger, Houssay, Yalow y muchos otros investigadores que han aportado nuevos conocimientos y tecnologías ${ }^{(6)}$.

\section{Diabetes en la antigüedad}

La primera referencia sobre la diabetes corresponde al papiro de Ebers (1500 años a.C.), con un párrafo de un sacerdote del templo del médico Inmhotep que habla de enfermos que adelgazan, que tienen hambre continuamente y orinan en abundancia, sintiéndose atormentados por la sed. Sin duda está describiendo los síntomas más graves de la diabetes tipo
$1^{(7-12)}$. Celso, describió una enfermedad consistente en poliuria indolora con emaciación. Areteo de Capadocia dio su nombre a la diabetes ("pasada a través de"); esta enfermedad se describe como una licuefacción de la carne y los huesos en la orina. Galeno habló de la diarrea urinaria, por un problema de "debilidad" renal en la que los líquidos se eliminaban sin cambio alguno. Los chinos hablaban de sed extrema, forunculosis y orina tan dulce que atraía a los perros. En la India era madhummeda o enfermedad de la orina de miel: Susruta escribió en el Ayur Veda que había dos tipos de pacientes con orina dulce, aquellos que tienen una tendencia congénita y aquellos en los que la enfermedad es propia de ricos, de obesos, de quienes comen mucho dulce y arroz; posiblemente ésta sea la primera descripción de la diabetes tipo 2. Avicena, Feliche y Paracelso tocaron el tema, a comienzos de la Edad Media.

\section{Edad Moderna}

En 1679, Tomás Willis humedeció su dedo en la orina de un paciente diabético, comprobando así su sabor dulce; por otro lado, encontró otros pacientes cuya orina no tenía ningún sabor y estableció entonces los términos de diabetes mellitus y diabetes insípida para diferenciarlos ${ }^{(13)}$.

El suizo Johann Conrad Brunner estuvo a punto de descubrir el origen pancreático de la diabetes en su libro Experimenta Nova circa Pancreas, en 1683. Al hacer incisiones en bazo y páncreas de perros de experimentación, manteniéndolos vivos y con su digestión normal, encontró que uno desarrolló extrema sed y poliuria, pero sin relacionar este hallazgo con la diabetes ${ }^{(14)}$.

Mathew Dobson descubrió, en 1775, que el sabor dulce era por la presencia de azúcar en la orina, lo que le permitió desarrollar después métodos de análisis para medir este glúcido.

John Rollo publicó sus observaciones sobre dos casos diabéticos (figura 2), describiendo muchos de los síntomas y el olor a acetona (que confundió con olor a manzana).

Figura 2. John Rollo y su publicación sobre diabetes

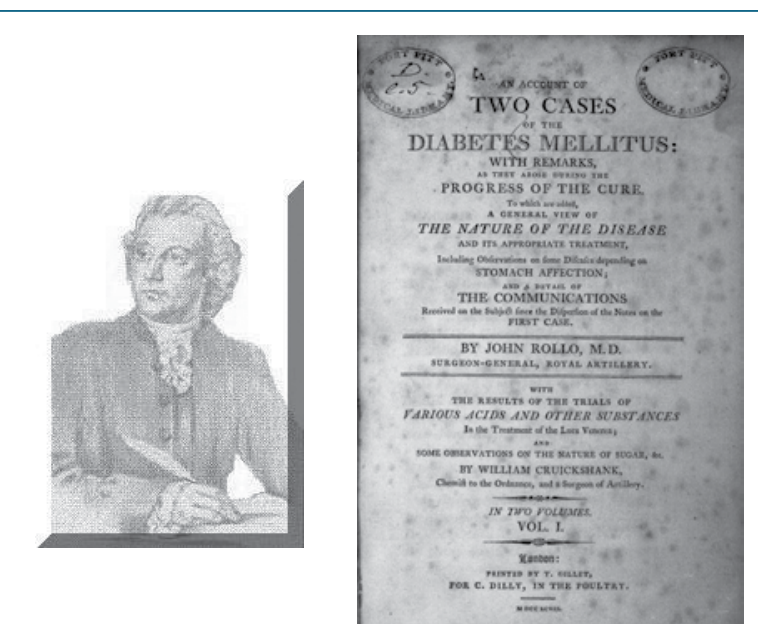




\section{Las dietas para el diabético}

Eran usadas en aquellos tiempos de manera circunstancial y sintomática para disminuir la glucemia y la glucosuria, restringiendo el aporte de carbohidratos. Precisamente Rollo propuso una dieta anorexiante -parecida a la actual de Atkins- observando que se reducía la glucemia y obteniendo una mejoría de la sintomatología en algunos $\operatorname{casos}^{(15)}$. Se utilizaron después la cura de cereales, la cura de arroz, la de leche, la de papas, la de avena y la de legumbres. Todas estas dietas empíricas se volvieron obsoletas después del descubrimiento de la insulina, cuando se diseñaron dietas balanceadas, e incluso dietas libres para los niños. Hasta la década de los 20, los diabéticos tenían pocas posibilidades de sobrevivir. Las dietas anoréxicas promovidas por el diabetólogo bostoniano Frederick M. Allen sólo conseguían prolongar pocos meses de vida.

En 1778, Thomas Cawley realizó la autopsia a un diabético y observó que tenía un páncreas atrófico y múltiples cálculos implantados en el tejido pancreático, esta es la primera referencia fundamentada que relaciona la diabetes mellitus y el páncreas $^{(7,12)}$.

\section{Siglo XIX}

Johann Peter Frank (1745-1821), de Gottingen, describió con exactitud la diabetes, distinguiendo la sacarina o verdadera de la insípida o falsa; se refirió a la disentería urinaria como el paso de bebidas no elaboradas a través de los riñones. En1806, Dupuytren afirmó que la albuminuria en el diabético es un signo de agravamiento de la enfermedad y en 1815 Chevreul identificó la glucosa como el azúcar presente en la orina de los diabéticos. En 1867, el estudiante de medicina Paul Langerhans descubrió en el páncreas de un mono unos islotes dispersos de células, con una estructura distinta de las células que producen los fermentos digestivos, cuya función es desconocida ${ }^{(12)}$.

En 1836, Ambrosiani encontró elevada la glucosa en la sangre de los diabéticos. En 1841, Trommer desarrolló una prueba para detectar azúcar en la orina. En 1848, Fehling detectó glucosuria con el licor que lleva su nombre

En 1852, Marchal de Calvi relató una observación en la cual señala la coincidencia de la gangrena con la diabetes. También habló después de la diátesis inflamatoria que, en situaciones de hiperglucemia sostenida, tiene una clara afinidad por la membrana interna de los pequeños vasos, por lo que, en suma, hoy conocemos como microangiopatía. Como el azúcar del diabético puede sufrir una fermentación alcohólica como se observó en dos casos con muerte imprevista, se comenzó a usar la levadura de cerveza en el tratamiento de la diabetes; esta hipótesis trataba de explicar los signos de excitación psíquica debidos a la cetoacidosis, síntomas comparables a los de la embriaguez alcohólica y a que el olor de la acetona puede ser confundido con el alcohol; también se manejó la hipótesis de que se tratase de una acidosis láctica inducida por el alco- hol. En 1864, dijo que la neuropatía en los diabéticos es secundaria a la enfermedad, describió las parestesias y dolores en estas neuropatías ${ }^{(16)}$.

En 1855, Jaeger -con el nuevo oftalmoscopio- describió la retinopatía en un diabético juvenil.

Claude Bernard (1813-1878) fue uno de esos médicos pensadores que modernizaron la enseñanza de la medicina e introdujeron las ciencias básicas en la educación. Se le considera como uno de los fundadores de la nueva ciencia de la endocrinología. En 1855, Bernard formuló los conceptos de secreción interna -mucho más amplio que el que se aceptó después- y de medio interno (ambiente fisiológico fijo de cada ser vivo) y la fisiología general, común a animales y vegetales. Bernard no sólo describió la glucogénesis hepática en perros alimentados con proteínas y azúcares sino que aisló el glucógeno del mismo órgano, observando además hiperglucemia después de la punción del cuarto ventrículo ${ }^{(17)}$.

1862. Pavy estableció una relación definida entre hiperglucemia y glucosuria, describió trastornos de la sudoración en diabéticos.

1866. Bouchardat propuso un origen pancreático de la diabetes; diseñó dietas balanceadas, fraccionadas e hipocalóricas en estos pacientes, al tiempo que señaló el valor del ejercicio físico en ellos ${ }^{(18)}$.

1869. Paul Langerhans, discípulo de Virchow, siendo aún estudiante, usando nuevas técnicas de tinción, describió nueve clases de células del páncreas del conejo, como parte de su tesis de grado ${ }^{(19)}$. Dentro de estas observó las de los islotes (figura 3), unas células pequeñas, poligonales, sin gránulos, que tenían el aspecto de manchas en el parénquima, aunque nunca conoció su función. Antes de esto había descrito unas células dendríticas epidérmicas no pigmentadas que, según se supo después, hacen parte del sistema inmune. También estudió el sistema macrófago.

Figura 3. Islotes de Langerhans (tinción hematoxilina-eosina)

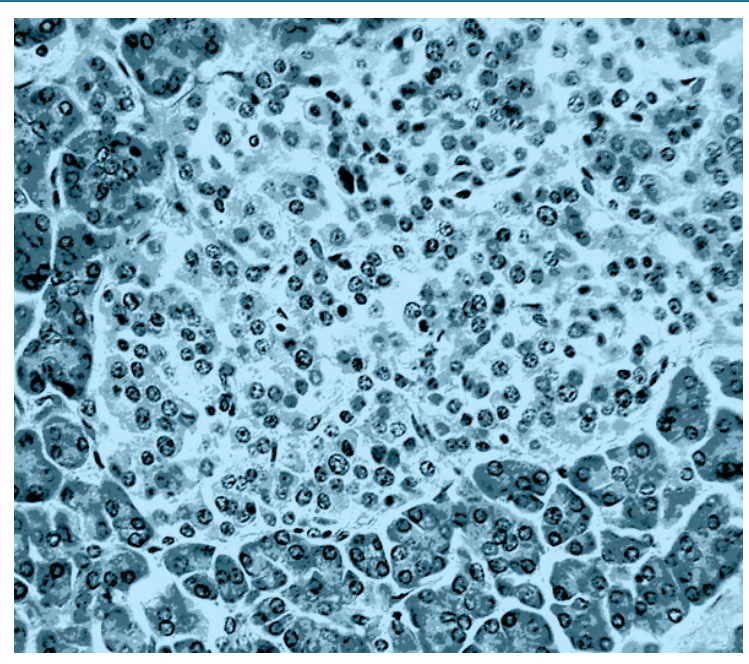


Otros descubrimientos relacionados con la diabetes también tuvieron lugar en la mitad del siglo XIX. Prout asoció el coma con la diabetes; Noyes observó que los diabéticos padecían de una forma de retinitis y, en 1874, Kussmaul (18221902) -que se recuerda por la respiración acidótica de los diabéticos en coma, con el clásico olor a manzana- hace la primera descripción completa del coma diabético.

1875. Naunyn introdujo el término acidosis para describir la fase aguda terminal de los diabéticos. Erhlich demuestra que los cambios renales en los diabéticos son debidos a la acumulación de glucógeno en los túbulos.

1877. Lancereux dice que hay dos tipos de diabetes: la grasa y la magra. En términos actuales podría referirse a los dos tipos principales de diabetes ${ }^{(20)}$.

1888. Giovanni Martinotti anunció en Turín que era perfectamente factible hacer pancreatectomías totales en pe$\operatorname{rros}^{(21)}$. Se afirma que el lituano Óscar Minkowski se basó en estas observaciones para practicar sus pancreatectomías.

1898. En Estrasburgo, el cirujano Oscar Minkowsky (figura 4), en el laboratorio de Bernard Naunyn y con el farmaceuta Joseph Von Mering como asistente, extirparon totalmente el páncreas de un perro para documentar los efectos de la ausencia de sus jugos; el animal se edematizó, presentó sed y poliuria. Encontraron sustancias reductoras en la orina, usando la prueba de

\section{Figura 4. Óscar Minkowski}

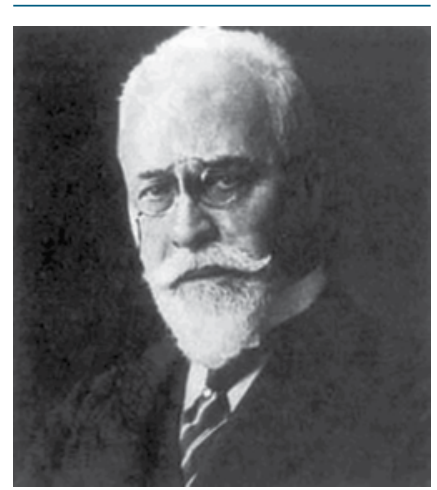

Trommer ${ }^{(22)}$. Minkowski -considerado el "abuelo" de la insulina- fue también el primero en describir la presencia del ácido betahidroxibutírico en el coma diabético ${ }^{(23)}$.
1889. Diamare sugiere que hay una relación entre los islotes pancreáticos y el metabolismo de los carbohidratos.

1893. Laguesse denominó los islotes pancreáticos con el nombre de quien los describió y planteó que producirían una secreción interna, aunque no mencionó su relación con la diabetes.

\section{Teorías patogénicas}

Antes del descubrimiento de la insulina, los médicos más conocidos postulaban sus diferentes teorías patogénicas de la diabetes. Bouchardat afirmaba que la diabetes se debía a un estado patológico del tubo digestivo. Unos promulgaban un origen pancreático, al no conseguir conciliar la conservación de funciones del páncreas exocrino con la disfunción del endocrino, acertada teoría sostenida por Lancereaux y Popper. Otros consideraban que la causa de la hiperglucemia es la producción exagerada de material glucogénico: teorías hematógena, miógena, histógena y nerviosa. También hubo quienes suponían una falta de utilización del azúcar normal por el organismo como Cantani y Bouchardat, entre otros.

Se mencionaban formas peculiares, fuente de interés y controversia en la época preinsulínica: la diabetes nerviosa (los nerviosos están más predispuestos a la enfermedad que los flemáticos), la diabetes conyugal o "contagiosa" (exposición de la pareja a influencias diabetógenas como la sífilis conyugal, alimentación inconveniente, excitaciones psíquicas y diabetes ligada al ejercicio profesional (es más frecuente la diabetes en las profesiones que exigen gran trabajo mental y causan alteraciones psíquicas; hay muchos eruditos, músicos, poetas, profesores, hombres de estado, grandes comerciantes y bolsistas $)^{(24)}$. El descubrimiento de la insulina ${ }^{(1)}$ y la revolución científica del siglo XX lograron la remoción de paradigmas equivocados con la práctica del método científico y con la utilización de tecnologías modernas.

\section{Referencias}

1. Barron M. The relation of the Islets of Langerhans to Diabetes. Surg Gynecol Obstet. 1920;31:437-448.

2. Bliss M. The discovery of insulin. Chicago: University of Chicago Press, 1982

3. Banting FG, Best CH, Collip JB, Cambell WR, Fletcher AA, Macleod JJR, Noble EC. The Effect Produced on Diabetes by Extractions of Pancreas. Transact Ass Amer Physicians. 1922; 37:337.

4. Banting FG, Best CH, Macleod JJR. The internal secretion of the pancreas. Am J Physiol. 1922;59:479.

5. Angelescu C, Paulescu NC. Omul şi opera sa medicală. Bucuresti: Vremea; 2009.

6. Rodríguez-Gama A. Premios Nobel de Medicina y Ciencias Fisiológicas. 1a. Edición 2012. Nomos Impresores, Bogotá.

7. Medvei VC. The History of Clinical Endocrinology: A Comprehensive Account of Endocrinology from Earliest Times to the Present Day. New York: Parthenon; 1993.

8. Amaro-Méndez S. Breve historia de la endocrinología. Editorial CientíficoTécnica, La Habana 1975.

9. Laìn-Entralgo P. Historia de la Medicina. la. Edición. Editorial Masson, Barcelona. 1978.

10. Lyons AS, Petrucelli RJ. Historia de la Medicina. 1a. Edición en español. Barcelona. Mosby/ Doyma Libros, 1994.

11. Jácome-Roca A. Historia de los medicamentos. Academia Nacional de Medicina, 2003, Bogotá.

12. Jácome Roca A. Historia de las Hormonas. Academia Nacional de Medicina, 2005. Bogotá.

13. Willis T. Opera Omnia. Coloniae: Sumptibus Gasparis Storti, 1694: 460. Leiden: Brill.

14. Brunner JC 1683 Experimenta nova circa pancreas. Wetstein, Amsterdam

15. Marble A. John Rollo. Diabetes. 1956 Jul-Aug;5(4):325-7.

16. Marchal de Calvi A.: Des rapports de la gangrène et de la glycosurie. Gaz Hôp Civ Mil 1852; 25: 178.

17. Ardila E. Claude Bernard y la medicina experimental. Rev Col Endocrinol Diab Metab 2015; 2 (3): 51-55

18. Bouchardat A. De la glycosurie ou diabète sucré. Son traitement hygiénique. Paris: Baillière, 1875.

19. Jolles S. Paul Langerhans. J Clin Pathol. 2002; 55(4): 243.

20. Lancereaux E. Notes et réflexions à propos de deux cas de diabètes sucré avec altération du pancréas. Bull Acad Méd Paris 1877; 6: 1215-1240.

21. Bruni B, Barbero PL, Blatto A. Giovanni Martinotti and experimental pancreatectomy, a century ago. Ann Osp Maria Vittoria Torino. 1989-1990; 31:203-14.

22. Brogard JM1, Vetter T, Blickle JF. Discovery of pancreatic diabetes in Strasbourg. Diabete Metab. 1992; 18(2):104-14.

23. Luft R. Oskar Minkowski: Discovery of the pancreatic origin of diabetes, 1889. Diabetologia 1989; 32 (7): 7399-401.

24. Medina Esteban JM. La patogenia de la diabetes mellitus en la era preinsulínica. Diario Médico, 2010. http://medicablogs.diariomedico.com/jena/2010/06/15. 